BULLETIN (New Series) OF THE

AMERICAN MATHEMATICAL SOCIETY

Volume 47, Number 4, October 2010, Pages 671-694

S 0273-0979(2010)01302-1

Article electronically published on July 20, 2010

\title{
STEADY WATER WAVES
}

\author{
WALTER A. STRAUSS
}

\begin{abstract}
We present a survey of certain aspects of the theory of steady water waves with emphasis on the role played by vorticity. Historical background, numerical illustrations, and brief discussions of the time-dependent problem and of approximate models are included as well.
\end{abstract}

\section{INTRODUCTION}

The motion of a fluid can be very complicated, as we know whenever we see waves break on a beach, fly in an airplane, or look at a lake on a windy day. The first comprehensive mathematical model of a fluid was proposed by Euler in the 1750s. It is amazing that Euler's equations and its variants are still the basic models used by mathematicians and engineers today. The most notable variant was introduced by Navier and Stokes to allow for the fluid to be viscous. Of course an immense amount of progress has been made over the past two and a half centuries. But one thing we know is that the difficulties of understanding fluids are so profound that there are huge problems that are still beyond our reach. This is well known both to mathematicians and to more practical scientists, such as hydraulic and aeronautical engineers. Euler [42] wrote, "If it is not permitted to us to penetrate to a complete knowledge concerning the motion of fluids, it is not to mechanics nor to the insufficiency of the known principles of motion that we must attribute the cause. It is analysis itself which abandons us here since all the theory of the motion of fluids has been reduced to the solution of analytic formulas". Euler's comment was amazingly prescient! In the many years since his time, the theory of fluids has had a tremendous effect on the historical development of mathematics itself. For instance, it was the main motivation for Cauchy to develop his complex function theory.

In this article I will concentrate on only one aspect of fluids, namely the theory of steady water waves, especially certain theoretical aspects where some recent progress has been made. There is no attempt to make either the contents or the references comprehensive.

Water flows are essentially incompressible. Water waves refer to the situation where the water lies below a body of air. The interface between the air and the water is a free surface. Free surfaces are mathematically challenging in their own right and they occur in many other situations such as the melting of ice (the free

Received by the editors August 19, 2009.

2010 Mathematics Subject Classification. Primary 76B15, 35Q31, 35R35.

I would like to thank my colleagues, Adrian Constantin, Joy Ko, and Samuel Walsh for their help with this paper, as well as the anonymous referees.

The work of this author was supported in part by NSF Grant DMS-0405066.

(c) 2010 American Mathematical Society Reverts to public domain 28 years from publication 
surface being the boundary of the ice) and the stretching of a flexible membrane over an obstacle (the free surface being the boundary of the contact region).

The Euler equations for an incompressible fluid are

$$
\nabla \cdot \mathbf{u}=0
$$

and

$$
\frac{\partial \mathbf{u}}{\partial t}+(\mathbf{u} \cdot \nabla) \mathbf{u}+\nabla P=\mathbf{F} .
$$

Here $\mathbf{x} \in \mathbb{R}^{3}$ is a point in space, $\mathbf{u}(\mathbf{x}, t)$ is the velocity of the fluid at the point $\mathbf{x}$ and time $t, P(\mathbf{x}, t)$ is the pressure, and $\mathbf{F}$ is an outside force that may be acting on the fluid (for instance, gravity). Although an incompressible fluid such as water can have variable density, we assume for simplicity that the density $=1$. Then the incompressibility is precisely expressed by (1.1). The balance of forces (Newton's Law) is expressed by (1.2), the first two terms representing the acceleration of a particle.

Now a water wave has a free boundary which is a surface $S$ in $\mathbb{R}^{3}$. We assume that the air is quiescent and $S$ is smooth: no bubbles or jets come in or out of the water. There are two boundary conditions. One expresses the fact that each water particle on $S$ remains on $S$ for all time; that is, particles of water do not invade the air, nor vice versa. The other simply states that on the surface the pressure $P$ equals the atmospheric pressure $P_{a t m}$ of the air. It is assumed that there is no surface tension (cf. Section 7).

Due to the great difficulty of the water wave problem, many approximate models have been studied, obtained by various scaling limits. The most famous one is KdV, but there are others such as the nonlinear Schrödinger equation and the Boussinesq systems (cf. Section 6).

What are some of the fundamental problems of interest to mathematicians?

(i) The Cauchy problem. That is, is there a unique solution for arbitrary initial conditions? For how long a time does it exist?

(ii) Are the solutions regular or do singularities develop after some time?

(iii) Are there solutions that are spatially periodic?

(iv) What is the effect of boundaries, for instance if the water sloshes against a dock or a ship or runs up a beach?

(v) What are the effects of viscosity? Of surface tension?

(vi) How can the various approximate models be justified?

(vii) What is turbulence and how can it be described?

(viii) Can we characterize the steady water waves?

Although there is an enormous literature on all of these problems, some of them are still in an elementary stage of development.

In Section 2 we introduce steady water waves, including some of the rich history of the subject. In Section 3 we provide a very brief survey of the initial-value problem for water waves. Section 4 is devoted to the existence theory of steady water waves with vorticity. The theory is illustrated by the numerical simulations of Section 5. In Section 6 we provide a very brief introduction to the various approximate models of water waves. This is followed in Section 7 by a long list of further problems relative to steady water waves. 


\section{2D PERIODIC STEADY WATER WAVES}

We first consider in some detail the case of special water waves satisfying the following six assumptions. Even with all these severe assumptions there are many open questions.

- The water is incompressible, without viscosity and without surface tension, so that the governing equations are as discussed above.

- It is two-dimensional. This means that the solutions are constant in one direction. That is, there is no motion in one of the horizontal directions. Think of a series of waves traveling in one direction as one often sees at the beach. We then write

$$
\mathbf{x}=\left(\begin{array}{l}
x \\
y
\end{array}\right), \quad \mathbf{u}=\left(\begin{array}{l}
u \\
v
\end{array}\right)=\left(\begin{array}{l}
u(x, y, t) \\
v(x, y, t)
\end{array}\right), \quad P=P(x, y, t) .
$$

We will regard $y$ as the vertical variable.

By the two-dimensional assumption the free surface $S$ is just a curve in the plane. Let us write it as $S(t)=\{y=\eta(x, t)\}$ and assume that $\eta$ is single-valued, nonnegative and smooth. Then the two boundary conditions mentioned above take the form

$$
P=P_{a t m}, \quad v=\eta_{t}+u \eta_{x} \quad \text { on } S .
$$

The vorticity $\boldsymbol{\omega}$ is the curl of the velocity: $\boldsymbol{\omega}=\nabla \times \mathbf{u}$. In our two-dimensional setting only the vertical component is nonzero, so we define the scalar vorticity as $\omega=v_{x}-u_{y}$. The other important scalar that can be defined because of the twodimensionality is the stream function defined by $\psi_{x}=-v, \psi_{y}=u$, due to (1.1), at least in a simply connected region. Then combining the last two definitions, we have

$$
\omega=v_{x}-u_{y} ; \quad \psi_{x}=-v, \psi_{y}=u ; \quad \omega=-\psi_{x x}-\psi_{y y} .
$$

We continue by listing the other assumptions.

- The only outside force is $\mathbf{F}=\left(\begin{array}{c}0 \\ -g\end{array}\right)$, where $g$ is the (constant) acceleration of gravity.

- The body of water has a fixed horizontal bottom $B=\{y=0\}$. Then on the fixed boundary $B$ there is just one boundary condition, namely, $v=0$.

- The wave is traveling (or "steady") at some constant speed $c>0$. That is, $\mathbf{u}$ and $P$ are functions of $(x-c t, y)$ while $\eta$ is a function of $x-c t$. Under this assumption we will go to a moving coordinate frame, changing $x-c t$ to $x$, whereby the time $t$ completely disappears.

- The wave is periodic in the horizontal variable $x$ with some period $L$.

One might think that with all these assumptions there are very few such waves. In fact, there are many of them. First let us mention a few facts about them. Details may be found in 22. The Euler equations imply that $\omega$ and $\psi-c y$ are functionally dependent, so that we get the elliptic PDE

$$
-\Delta \psi(x, y)=\gamma(\psi(x, y)-c y)=\omega(x, y)
$$

from (2.2), where $\Delta \psi=\psi_{x x}+\psi_{y y}$. The vorticity function $\gamma$ is a completely arbitrary function of one variable $(\psi-c y)$; we assume it is single-valued. There are two constants associated with any such wave. First, the flux

$$
p_{0}=\int_{0}^{\eta(x)}[u(x, y)-c] d y
$$


is independent of $x$. Second, the relative stream function $\psi(x, y)-c y$, restricted to the free surface $S$, is a constant. The complete problem can be expressed as

$$
\begin{gathered}
\Delta \psi=\gamma(\psi(x, y)-c y) \text { in }\{0 \leq y \leq \eta(x)\}, \quad \psi=-p_{0} \text { on }\{y=0\}, \\
\psi-c y=0 \quad \text { and }|\nabla(\psi-c y)|^{2}+2 g(y+d)=Q \quad \text { on } S=\{y=\eta(x)\},
\end{gathered}
$$

where $Q$ is a constant of integration.

The study of such waves goes back over two centuries. Most of the work on this problem over all those years assumed that the flow is irrotational, that is, $\omega=0$, whence (2.3) implies that $\psi$ is a harmonic function. Already Newton had attempted a theory of water waves, but the big breakthrough came when Euler proposed his general equations for a fluid.

Laplace in 1776 and Lagrange in 1781 were the first to make progress on water waves. Lagrange formulated the equations of mechanics, and in particular water waves, in terms of variables that are labels for the particles rather than for the points in space. This is the Lagrangian formulation. Then, and for a long time thereafter, most of the work was concerned with the linearized theory, that is, for waves of small amplitude. In a prize competition in 1815, the young Cauchy (25 years old) made a major advance. Although it was not published until 1827, it appears as the first paper in Volume 1 of his Oeuvres Complètes [15]. Poisson, who was on the jury of the competition, independently had similar results. The method that both of them brought to bear on the linearized problem of infinite depth was what we now call the Fourier transform. Fourier himself was a young contemporary who concentrated mainly on the problem of heat conduction and apparently did not concern himself with fluids. Nevertheless, Cauchy and Poisson must have had some knowledge of Fourier's early unpublished work. Paris in the early years of the 19th century must have been a cauldron of mathematical activity. Cauchy was concerned to a great extent with the two-dimensional case. The techniques he developed for water waves were the primary motivation for his function theory, which was first properly developed from the point of view of complex quantities in 1825. For more on the history, see [33, 36, 38, 39.

Analysis of the irrotational (zero vorticity) case continued throughout the 19th century. In this case, $\Delta \psi=0$ and therefore the interior of the fluid is simply described by a harmonic function. Then a conformal map transforms the problem to a fixed domain, whence by means of a Green's function it can be reformulated as an integral equation on the boundary. For instance, Airy [1] derived the dispersion relation for the wave velocity in the linear approximation: $c^{2} k=g \tanh (k d)$, where $2 \pi / k$ is the period of the wave and $d$ is the depth. Stokes [78 made many contributions, for instance observing that crests tend to be sharper and troughs flatter as the amplitude increases, and that the crest of a steady wave of greatest possible height is a stagnation point with a $120^{\circ}$ corner.

It was not until the 1920s that rigorous constructions were made of exact nonlinear steady periodic irrotational water waves of infinite depth. The first results were by Nekrasov [70] in Russia and Levi-Civita [65] in Italy, independently, who considered the amplitude $a$ to be a small parameter, expanded the presumed solution in powers of $a$ and proved that there is a positive radius of convergence. Struik, who was a postdoc of Levi-Civita, extended the work to the case of finite depth. Much later Krasovskii [62, Keady and Norbury [57, and Amick and Toland [5] were finally able to construct large amplitude waves and to prove rigorously Stokes' $120^{\circ}$ 
conjecture at the crest. All of this work strongly used the assumption that the flow is irrotational, whereby $\psi$ is a harmonic function inside the fluid. Some excellent modern surveys of (mostly) irrotational steady water waves are [79, 71, 40, 48, 32.

It is true that in the absence of initial vorticity, boundaries or currents, a water wave will have no vorticity at all future times. On the other hand, rotational effects are significant in many circumstances, for instance for wind-driven waves, waves riding upon a sheared current, or waves near a ship or pier. In 1802, Gerstner 47. (see also [16) produced a remarkable example of a steady periodic water wave with infinite depth and nonzero vorticity. It is an exact solution and is rigorously correct. This was possible 200 years ago because in Lagrangian coordinates it is expressible in terms of sines and cosines. In fact, if the position of a water particle in Gerstner's flow is labeled by $(a, b)$, then its position at time $t$ is

$$
x=a+\frac{e^{m b}}{m} \sin (m a+\sqrt{g m} t), \quad y=b-\frac{e^{m b}}{m} \cos (m a+\sqrt{g m} t),
$$

where $-\infty<b \leq b_{0} \leq 0$ and $m$ is any positive constant. It is an exact solution of infinite depth. The velocity of course is $u=\frac{d x}{d t}, v=\frac{d y}{d t}$. The free surface $S$ is given by this formula with $b=b_{0}$, where $b_{0}$ is any nonpositive constant. If $b_{0}<0, S$ is a trochoid; if $b_{0}=0, S$ is a cycloid. Clearly, the individual water particles travel in circles and their radii become smaller with depth. No exact solution formula of this type is known in the case of finite depth.

For the case of vorticity the existence of large classes of small-amplitude solutions was proven by Dubreil-Jacotin [41] in 1934 and large-amplitude solutions by Constantin and Strauss [22]. This situation requires looking inside the fluid because $\psi$ is no longer a harmonic function so that it is no longer viable to use conformal mapping.

\section{Cauchy problem}

The Cauchy problem for the Euler equations is to solve (1.1), (1.2) for an arbitrary initial condition $\mathbf{u}(\mathbf{x}, 0)=\mathbf{u}_{0}(\mathbf{x})$ at $t=0$. It is assumed that $\nabla \cdot \mathbf{u}_{0}=0$. The simplest case is to take $\mathbf{F}=\mathbf{0}$ and $\mathbf{x} \in \mathbb{T}^{n}$, the $n$-torus, which is of course equivalent to periodicity in each coordinate. If we take the divergence of (1.2) and make use of (1.1), we find that

$$
\sum\left(\partial_{i} u_{j}\right)\left(\partial_{j} u_{i}\right)+\Delta P=0,
$$

where $\partial_{i}=\partial / \partial x_{i}$. Thus the pressure is determined by the velocity (and the boundary conditions) at each time and so it usually plays a subsidiary role in the analysis.

Short-time existence for the Euler and Navier-Stokes equations in a fixed domain such as $\mathbb{T}^{n}$ is well known: there are hundreds of papers on this subject that treat various different function spaces. The modern theory was initiated by Leray [64. Global existence, that is, the problem of solving the equations for all times $0 \leq t<$ $\infty$ is much more subtle. In two dimensions the problem was solved for the Euler equations in several stages since the 1930s but in three dimensions it is still open. For the Navier-Stokes case, with its addition of the (regularizing) term $\nu \Delta \mathbf{u}$, where $\nu$ is the viscosity coefficient, it is one of the "millennium" problems 43 . 
The water wave problem (for Euler) with its free boundary is yet more difficult. One now provides both the initial velocity $\mathbf{u}_{0}$ and the initial free surface $S_{0}$. Both the velocity and the surface evolve in time; the pressure is then given by (3.1). It was not until recently that $\mathrm{Wu}[86,87$ ] proved that arbitrary irrotational initial data in a Sobolev space lead to short-time existence. Since then there has been a great deal of activity on the short-time problem with increasing generality (permitting vorticity, compressibility, surface tension, uneven bottoms, etc.). These papers use a variety of techniques, some using Eulerian and others Lagrangian coordinates, some using the Nash-Moser method and others simple iteration, some emphasizing a geometric or a variational point of view. Some of the key papers are [3, 4, 26, 63, 69, 68, 75, 77, 91.

In the irrotational case, for which $\partial_{i} u_{j}=\partial_{j} u_{i}$, (3.1) implies that $\Delta P \leq 0$, which implies by the maximum principle that $P$ is minimized on the boundary. If the minimum occurs on the free surface $S$, then $\partial P / \partial n<0$ there, where $\mathbf{n}$ is the outward normal. However, in the rotational case (i.e., with vorticity) this inequality is assumed on $S$; it is called the Rayleigh-Taylor stability condition. The Rayleigh-Taylor instability refers to the opposite case when there are two fluids, irrotational or not, a heavier one on top of a lighter one, so that singularities are expected to form as the heavier fluid penetrates the lighter one and tends to create "fingers". It is expected that surface tension moderates the instability and makes the solution more regular. A closely related problem is when the two fluids are separated by a free interface and move past each other at different velocities so that there is vorticity along the interface. Then spiral waves are expected to form along the interface; this is called the Kelvin-Helmholtz instability.

After a long time it is expected that turbulence is likely to develop in a fluid. However, if the initial data are very small, it should take a very long time. Very recently there has been mathematical progress on the long-time behavior if one assumes that the initial data are small. Wu 88 , proves in two dimensions that there exists an $\epsilon_{0}>0$ such that if the irrotational initial data have size $\epsilon<\epsilon_{0}$ in a certain Sobolev norm, then the solution exists in a long time interval $0 \leq t<\exp (C / \epsilon)$ for some constant $C$. This is called "almost-global" existence. Germain, Masmoudi and Shatah [46] and $\mathrm{Wu}\left[89\right.$ ] prove in three dimensions that there exists $\epsilon_{0}>0$ such that if the irrotational data $\mathbf{u}_{0}$ has size less than $\epsilon_{0}$ in a certain Sobolev norm, then the solution exists for all time $0 \leq t<\infty$. For instance, in [46], a sufficient condition is

$$
\sum_{|\alpha| \leq N} \int\left\{\left|\partial^{\alpha} \mathbf{u}_{0}\right|+\left|\partial^{\alpha} \mathbf{u}_{0}\right|^{2}\right\} d x+\int|x|^{2}\left|\mathbf{u}_{0}\right|^{2} d x<\epsilon_{0}
$$

for some sufficiently large $N$. (The actual condition in both papers is better than this.) Both proofs use the method of normal forms [76 together with the method of vector fields [58, but otherwise the proofs are quite different. The key ingredients of the proof in [46] are: (i) high-order energy estimates and (ii) dispersive estimates which are obtained from the analysis of the spatial and temporal resonances. A key ingredient of the proof in [89] is the use of analytic theory in the Clifford algebra framework. In general, higher dimensions have a certain advantage in this type of small-amplitude problem because the basic decay rate in time for the linearized equation is faster, which contributes to improved dispersive estimates. 


\section{EXISTENCE OF PERIODIC STEADY WAVES}

Resuming the discussion of Section 2, we have the following existence theorem.

Theorem 4.1 (22). Let arbitrary constants $c>0, L>0, p_{0}<0$ (wave speed, period, flux) be given as well as an arbitrary function $\gamma(\cdot) \in C^{1, \alpha}$ (for some $0<$ $\alpha<1$ ) subject to a "bifurcation condition" (see just below (4.6)).

Then there exists a connected set $\mathcal{C}$ of traveling waves with $u(x, y)<c$ in the function space $\mathbf{u} \in C^{2, \alpha}, \eta \in C^{3, \alpha}$, symmetric around the crest. There is exactly one crest and one trough per period. The set $\mathcal{C}$ contains exactly one wave with $S$ flat and with all motion horizontal (a "laminar flow"), as well as a sequence of waves for which $\max u \nearrow c$ ("flows approaching stagnation").

The bifurcation condition (stated below (4.6)) is satisfied if either $\gamma \leq 0$ or $L$ is not too small or $\left|p_{0}\right|$ is not too big. The fact that $\gamma \leq 0$ implies the bifurcation condition is proven by Wahlén 82. The waves near the laminar flow (small-amplitude waves) look like cosine waves. What do the waves that are far along the "continuum" $\mathcal{C}$ look like? The theory does not provide much information. So we performed some numerical computations [59]. We chose $L=2 \pi, p_{0}=-2$ and $g=9.8$. Figure 1 provides an example.

Figures 2 and 3 compare waves with different constant vorticities. For each of these waves the crest is near stagnation. A point of stagnation is a point $(x, y)$ at which $u=c$ and $v=0$.

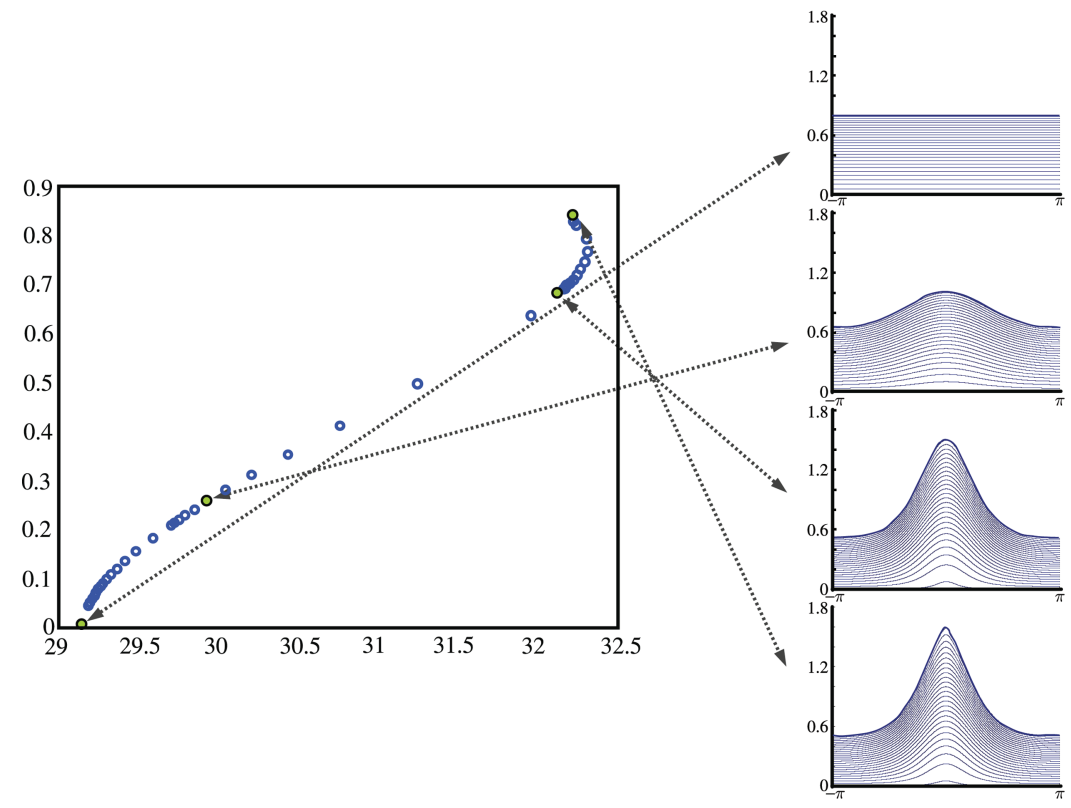

Figure 1 . The continuum $\mathcal{C}$ : On the left, amplitude vs. energy for $\gamma \equiv 2.95$. Stagnation occurs at the crest for the wave at the upper end of $\mathcal{C}$. On the right are four of the waves together with some of their streamlines over a single period. 


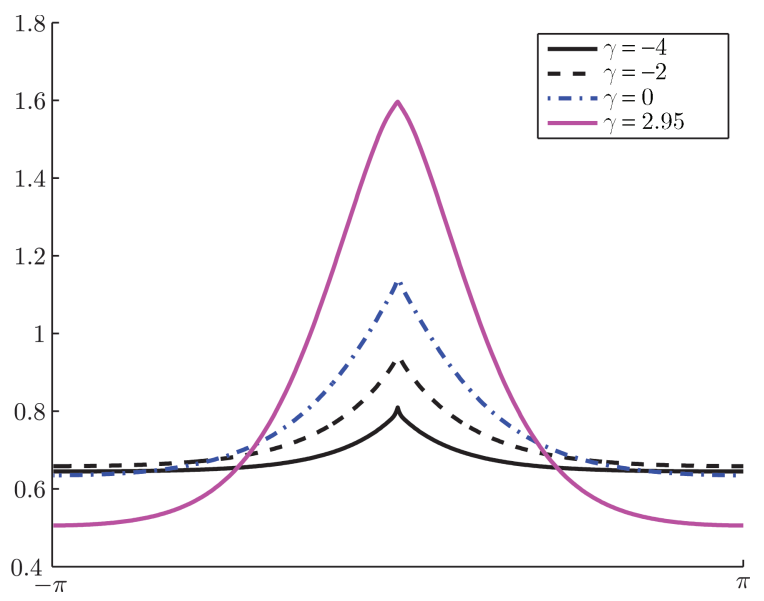

Figure 2. Profiles of waves near stagnation compared for four values of constant vorticity: $\gamma \equiv-4,-2,0,2.95$. This figure shows that the vorticity, even if constant, has a big qualitative effect on the solutions!

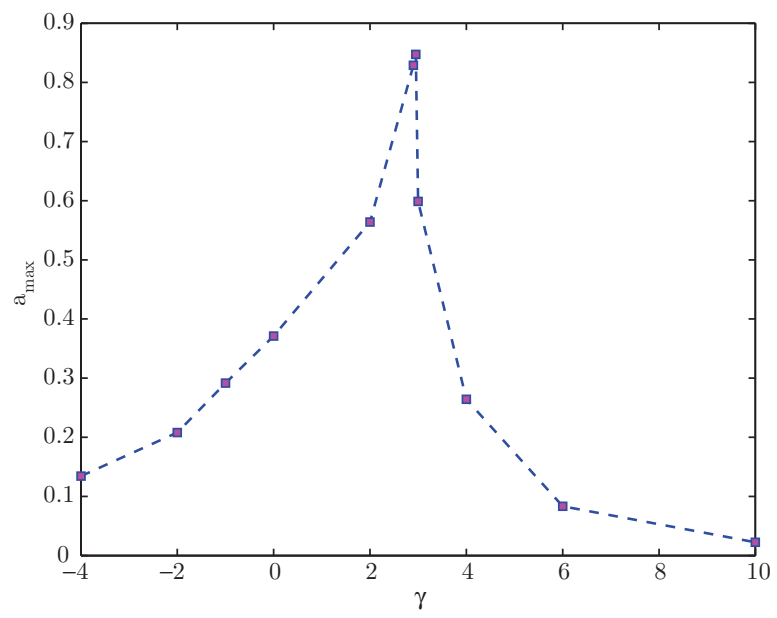

Figure 3. Maximum amplitude along $\mathcal{C}$ vs. constant vorticity. The switchover from stagnation at the crest to stagnation at the bottom point below the crest occurs between $\gamma=2.95$ and $\gamma=$ 3.00 , exactly where the maximum amplitude starts to decrease.

The same methods used to prove the existence theorem are used to perform the simulations. The key idea, as in all free boundary problems, is to fix the free boundary. There are at least three ways to do it.

(1) The flattening transformation $y \rightarrow \frac{y}{\eta(x)}$ leads to a rather messy set of equations, which can sometimes be useful for small-amplitude waves.

(2) The conformal transformation

$$
p=c y-\psi(x, y), \quad q=\text { its harmonic conjugate, }
$$


uses the fact that $p=c y-\psi(x, y)$ is constant on the free surface and on the bottom. Its advantage is that the Laplacian remains the same in the new variables up to a factor. However, it requires $\psi$ to be harmonic and so is valid only in the irrotational case.

(3) The Dubreil-Jacotin transformation

$$
p=c y-\psi(x, y), \quad q=x,
$$

uses the same fact. Its advantage is that it permits arbitrary vorticity. Its disadvantage is that it requires $u<c$. See Figure 4 .

The D-J transformation (4.1), together with the height $h(q, p)=y$ as dependent variable, is simply an exchange of independent and dependent variables. An elementary calculation shows that it leads to the following system in the rectangle $R=\left\{-L / 2<q<L / 2, p_{0}<p<0\right\}$ :

$$
\begin{aligned}
& \left(1+h_{q}^{2}\right) h_{p p}-2 h_{q} h_{p} h_{q p}+h_{p}^{2} h_{q q}+\gamma(-p) h_{p}^{3}=0 \quad \text { in } R, \\
& 1+h_{q}^{2}+(2 g h-Q) h_{p}^{2}=0 \quad \text { on the top }(T=\{p=0\}), \\
& h=0 \quad \text { on the bottom }\left(B=\left\{p=p_{0}\right\}\right), \\
& \text { period } L \text { in the } q \text { variable. }
\end{aligned}
$$

The constant $Q$ enters into (4.3) as a constant of integration. Notice that the quadratic form $\left(1+h_{q}^{2}\right) \alpha^{2}-2 h_{q} h_{p} \alpha \beta+h_{p}^{2} \beta^{2}$ coming from the PDE (4.2) is positive definite so long as $h_{p}>0$. This means that the PDE is elliptic for $h_{p}>0$. The change of variables implies that $h_{p}=1 /(c-u)$, so that this condition means $u<c$.

Thus the whole problem has been transformed into (4.2)-(4.5) provided $u<c$. Notice that the only free parameters are the constant $Q$, the height $\left|p_{0}\right|$ and width $L$ of the rectangle, and the vorticity function $\gamma(\cdot)$. The time $t$ as well as the wave speed $c$ have disappeared. The constant $Q$ is essentially the energy and is sometimes

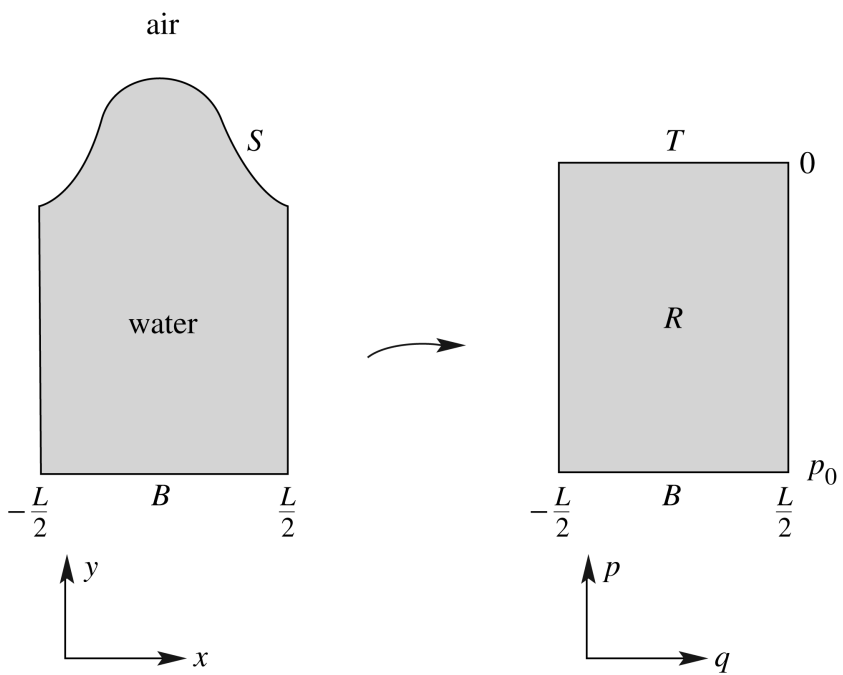

Figure 4. The DJ transformation 
called the hydraulic head. Going back to the original variables,

$$
Q=\frac{1}{2}(c-u)^{2}+\frac{1}{2} v^{2}+g y+P-\Gamma(c y-\psi)
$$

everywhere in the fluid region, where $\Gamma^{\prime}(p)=\gamma(-p)$. It can be proven that for arbitrary $c$ every solution of (4.2)- (4.5) can be transformed back to a solution of the original problem 22 .

Sketch of Proof of Theorem 4.1, We work entirely with the transformed system (4.2) - (4.5) and make use of bifurcation theory, beginning with the local theory. We treat $Q$ as a bifurcation parameter, while $\gamma(\cdot)$ is arbitrary but fixed.

First, it is elementary to see that there is a curve of solutions of (4.2)-(4.5) that are independent of $q$. In that case, (4.2) becomes an ODE of second order, (4.4) fixes one of the constants of integration, and (4.3) simply provides a relation between $Q$ and the other constant $\lambda$. These laminar (i.e. flat) solutions form a curve in function space, the "trivial" curve. Let us denote them as $(Q(\lambda), H(p ; \lambda))$, where $H(p ; \lambda)=\int_{p_{0}}^{p}(\lambda+2 \Gamma(s))^{-1 / 2} d s$. We consider them in the $(Q, h)$ space $\mathbb{R} \times C^{3, \alpha}(\bar{R})$, where $0<\alpha<1$.

We search along this trivial curve for a bifurcating curve $\mathcal{C}_{\text {loc }}$ of nontrivial solutions. This could occur only at a point $\left(Q^{*}, H^{*}\right)=\left(Q\left(\lambda^{*}\right), H\left(p, \lambda^{*}\right)\right)$ where the linearized operator has a nontrivial kernel. The linearized problem is

$m_{p p}+H_{p}^{2} m_{q q}+3 \gamma(-p) H_{p}^{2} m_{p}=0 \quad$ in $R, \quad m_{p}=\lambda^{-3 / 2} g m$ on $T, \quad m=0$ on $B$,

together with periodicity in $q$. The parameter $\lambda$ appears in three places in this "eigenvalue" problem. Our "bifurcation condition" is that (4.6) has a solution of the form $m=M(p) \cos (2 \pi q / L)$ with $M \not \equiv 0$. (More generally, we could take $\cos (k 2 \pi q / L)$ for any integer $k$.) Even though this is not a standard eigenvalue problem, it is still possible to apply the Crandall-Rabinowitz theorem [34] on bifurcation from a simple eigenvalue (or the Liapunov-Schmidt method) to get a unique local curve of nontrivial solutions. The amplitudes of all the waves so constructed are small.

In order to continue the curve beyond the local region near the bifurcation point, we use infinite-dimensional degree theory. Such a degree was pioneered by Leray and Schauder in the 1930s. Because of the nonlinear boundary condition, we have to use the more general degree constructed by Healey and Simpson [50]. A convenient space in which to work is $\mathbb{R} \times C^{3, \alpha}(\bar{R})$. The key feature of this space is the classical Schauder estimate, which provides an estimate of a solution of a second-order linear elliptic equation in a space $C^{k+2, \alpha}$ in terms of the right-hand side in $C^{k, \alpha}(k=$ $0,1,2, \ldots)$.

Generalizing the global bifurcation method of Rabinowitz [73], we will show that there is a connected set $\mathcal{C} \subset \mathbb{R} \times C^{3, \alpha}(\bar{R})$ such that only the following alternatives remain (see Figure 5):

(1) Unboundedness: $\mathcal{C}$ becomes unbounded.

(2) Self-intersection: $\mathcal{C}$ intersects the trivial curve at another point.

(3) Degeneracy: The PDE becomes nonelliptic or the BC becomes nonoblique. 


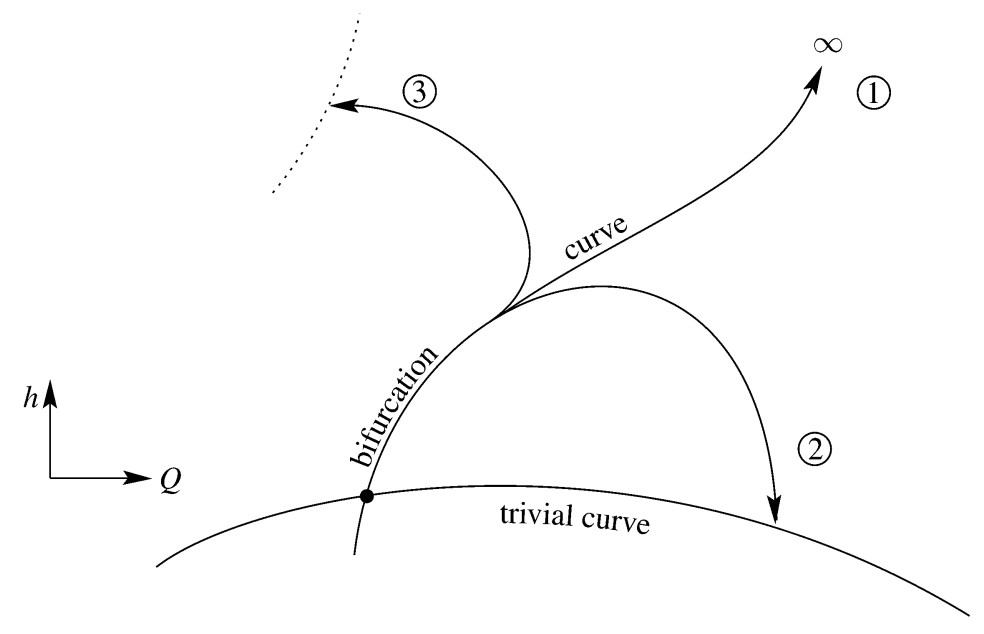

FIGURE 5. Sketch of the three alternatives.

To prove this, we define operators $\mathcal{G}_{1}$ and $\mathcal{G}_{2}$ so that (4.2), (4.3) take the form

$$
\mathcal{G}_{1}(h)=0 \text { in } R, \quad \mathcal{G}_{2}(Q, h)=0 \text { on } T
$$

and we write $\mathcal{G}=\left(\mathcal{G}_{1}, \mathcal{G}_{2}\right)$. This is a quasilinear elliptic equation with a fully nonlinear boundary condition. Define the spaces

$$
X=\left\{h \in C^{3, \alpha}(\bar{R}) \mid h=0 \text { on } B\right\}, \quad Y=C^{1, \alpha}(\bar{R}) \times C^{2, \alpha}(T)
$$

with periodicity and symmetry in the horizontal variable $q$. For a fixed small $\delta>0$, define

$$
\mathcal{O}_{\delta}=\left\{(Q, h) \in \mathbb{R} \times X \mid h_{p}>\delta \text { in } \bar{R} ; 2 g h<Q-\delta \text { on } T\right\}
$$

and define $\mathcal{C}_{\delta}$ to be the component containing $\left(Q^{*}, H^{*}\right)$ of the closure in $\mathbb{R} \times X$ of $\left\{(Q, h) \in \mathcal{O}_{\delta} \mid \mathcal{G}(Q, h)=0 ; h_{q} \not \equiv 0\right\}$. The inequalities enforce uniform ellipticity in $\bar{R}$ and uniform obliqueness on $T$.

The Healey-Simpson degree is defined for Banach spaces $X, Y$ and a map $\mathcal{F}$ : $\mathcal{O} \mapsto Y$ with an open set $\mathcal{O} \subset X$ and a point $y \in Y$ with $y \notin \mathcal{F}(\partial \mathcal{O})$. The mapping $\mathcal{F}$ must satisfy appropriate conditions, notably some compactness. See [50] for details. For regular values $y \notin \mathcal{F}(\partial \mathcal{O})$,

$$
\operatorname{deg}(\mathcal{F}, \mathcal{O}, y)=\sum_{\mathcal{F}(h)=y, h \in \mathcal{O}}(-1)^{\nu(h)}
$$

where $\nu(h)$ is the number of positive real eigenvalues $\kappa$, counting multiplicity, of the Fréchet derivative, which is a linear operator $\mathcal{F}^{\prime}(h)$. The regular values are dense by the Sard-Smale theorem.

Now suppose that $\mathcal{C}_{\delta}$ is bounded in $\mathbb{R} \times X$, does not contain any other laminar solution, and does not meet the boundary of $\mathcal{O}_{\delta}$. We will obtain a contradiction as follows. We cover the compact set $\mathcal{C}_{\delta}$ by a slightly bigger open set $\mathcal{P}$. For each $Q$ we excise a small ball $B_{Q}$ around the laminar solution and look at the degree over the three sets $B_{Q} \cup\left(\mathcal{P}_{Q} \backslash B_{Q}\right)=\mathcal{P}_{Q}$. The degree satisfies the addition property

$$
\operatorname{deg}\left(\mathcal{G}(Q, \cdot), B_{Q}, 0\right)+\operatorname{deg}\left(\mathcal{G}(Q, \cdot), \mathcal{P}_{Q} \backslash B_{Q}, 0\right)=\operatorname{deg}\left(\mathcal{G}(Q, \cdot), \mathcal{P}_{Q}, 0\right) .
$$


As $Q$ moves past $Q^{*}$, the simple eigenvalue changes sign, so that $\nu(h)$ changes by one and the first of the three degrees in (4.7) also changes sign. The second of these degrees vanishes for $Q \neq Q^{*}$ by homotopy invariance because it vanishes for large $|Q|$. The third of these degrees is independent of $Q$, for $Q$ near $Q^{*}$, by homotopy invariance since $\mathcal{G} \neq 0$ on $\partial\left(\mathcal{P}_{Q}\right)$. Thus (4.7) is contradicted. Hence either $\mathcal{C}_{\delta}$ is unbounded in $\mathbb{R} \times X$, or it meets the trivial curve elsewhere, or it meets the boundary of $\mathcal{O}_{\delta}$. (The $\delta$ will be removed below.)

The self-intersection alternative is now eliminated by using the nodal properties of the waves, that is, proving that the free surface $S$ is strictly monotone between crest and trough. Indeed, we prove that $h_{q}<0$ from crest to trough (inside the fluid and on the free surface) as well as $h_{q q}<0$ at the trough and $h_{q q}>0$ at the crest, and this is valid for all the waves in $\mathcal{C}_{\delta}$. The proof 22, 23, is based on the maximum principles of Hopf and Serrin [44. Then the possibility that $\mathcal{C}_{\delta}$ might return to the trivial curve at some other point $\left(Q^{* *}, H^{* *}\right)$ could not occur because $Q^{* *}$ would correspond to a different "eigenvalue" with the wrong nodal property. Now the alternative that $\mathcal{C}_{\delta}$ is unbounded could occur either if $Q$ is unbounded in $\mathbb{R}$ or if $h$ is unbounded in $X \subset C^{3, \alpha}$. By use of the Lieberman-Trudinger regularity estimates 66], this is reduced to the much weaker statement that either $Q$ is unbounded or merely $\sup \left|h_{p}\right|$ is unbounded.

We now define the full continuum as $\mathcal{C}=\bigcup_{\delta>0} \mathcal{C}_{\delta}$. All that remains are the alternatives that either sup $\left|h_{p}\right|$ is unbounded, or $Q$ is unbounded, or $h_{p} \rightarrow 0$, or $Q-\left.2 g h\right|_{T} \rightarrow 0$ somewhere along $\mathcal{C}$. Recalling that $h_{p}=1 /(c-u)$, we see that the first of these possibilities implies stagnation. It is not difficult to see that all four possibilities imply that either $\sup u \nearrow c$ or inf $u \searrow-\infty$ somewhere along $\mathcal{C}$. Next we prove two lemmas. One gives a lower bound on $u$ at the crest all along $\mathcal{C}$. The other one, which is based on the superharmonic inequality $\Delta P \leq \gamma^{2}(\psi)$, provides the inequality that $P-\mathcal{P}_{a t m}$ is at least $\sup \gamma^{2} / 2$ times the height of the crest. Using these two lemmas and the fact that $p_{0}$ is fixed, it follows that inf $u \searrow-\infty$ implies $\sup u \nearrow c$.

More generally, if $\gamma \in C^{k, \alpha}$ for some integer $k \geq 1$, then $\mathbf{u} \in C^{k+1, \alpha}, \eta \in$ $C^{k+2, \alpha}, P \in C^{k+2, \alpha}$. Thus if $\gamma$ is $C^{\infty}$, so are all the waves in $\mathcal{C}$. The statement about the pressure follows from the elliptic equation $P_{x x}+P_{y y}=2 \psi_{x x} \psi_{y y}-2 \psi_{x y}^{2}$.

Furthermore, there exists a continuous curve $\mathcal{K} \subset \mathcal{C}$ that contains both a laminar wave and a sequence of waves approaching stagnation. This is proven by Walsh 84 by means of the method of real-analytic continuation in Banach spaces of Dancer [37. (see also [14]) rather than by means of degree theory.

In most of the computations of the next section we take the vorticity $\gamma(\cdot)$ to be a step function, for the sake of conceptual simplicity. Such a $\gamma$ is therefore excluded from Theorem 4.1, This gap is filled by the following theorem [21]. The transformed PDE (4.2) is rewritten in the weak form

$$
\left\{-\frac{1+h_{q}^{2}}{2 h_{p}^{2}}+\Gamma(p)\right\}_{p}+\left\{\frac{h_{q}}{h_{p}}\right\}_{q}=0
$$

where $\Gamma$ is defined above. Let $c, L, p_{0}$ be given as in Theorem 4.1. Let $0<\alpha<1$. If $\gamma$ is a step function on $\left[p_{0}, 0\right]$, then Theorem 4.1 is valid with $\mathbf{u} \in C^{\alpha}$ and $\eta, P, h \in C^{1, \alpha}$. Of course the derivatives are taken in the sense of distributions. 


\section{Simulation}

The steady waves can be simulated numerically using the following steps.

- Find the bifurcation point numerically.

- Discretize with a finite-difference scheme.

- Use a nonlinear solver (Newton method).

- Employ numerical continuation along the continuum $\mathcal{C}$. (We used the continuation library LOCA in the software package TRILINOS.)

- Continue until almost-stagnation $(u \sim c)$, leading to waves of large amplitude.

The vorticity is arbitrary. One interesting scenario is for a vorticity layer at the bottom, which models a current such as an undertow (see 60]). Another scenario is wind on the surface. It is known experimentally that wind creates shear (that is, vorticity) near the surface. Therefore we decided to perform simulations with a shear layer near the surface. Negative vorticity means intuitively that the infinitesimal rotations go clockwise in the $(x, y)$-plane, which corresponds to a favorable wind, that is, going in the same direction as the surface wave (to the right under our convention $c>0$ ). Similarly, positive vorticity corresponds to counterclockwise rotations and an adverse wind. It turns out, not surprisingly, that a wind that is adverse can produce steady waves of higher amplitude than a favorable wind. The following simulations taken from 60 illustrate nearly stagnant waves for the case of a surface layer of vorticity of thickness $\theta \%$ and vorticity strength $\gamma_{0}$, irrotational below the layer. By this we mean that the vorticity equals $\gamma_{0}$ in the region $\left\{(x, y): 0<\psi(x, y)-c y<\frac{\left|p_{0}\right| \theta}{100}\right\}$ just below $S$. As before, $L=2 \pi, g=9.8, p_{0}=-2$. See Figures 6 9] Finally, in Figure 10 we include a simulation of a continuous vorticity distribution. In this example, the vorticity is $\gamma(-p)=10 \tanh 10(1+p)$ for $0<-p<2, \quad p=-\psi+c y$.

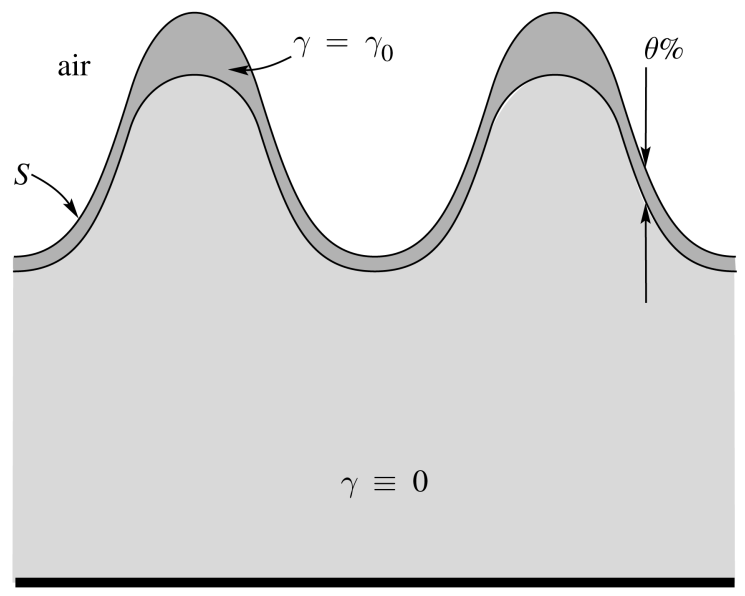

FiguRE 6. Rough sketch of a vorticity layer near the free surface. 

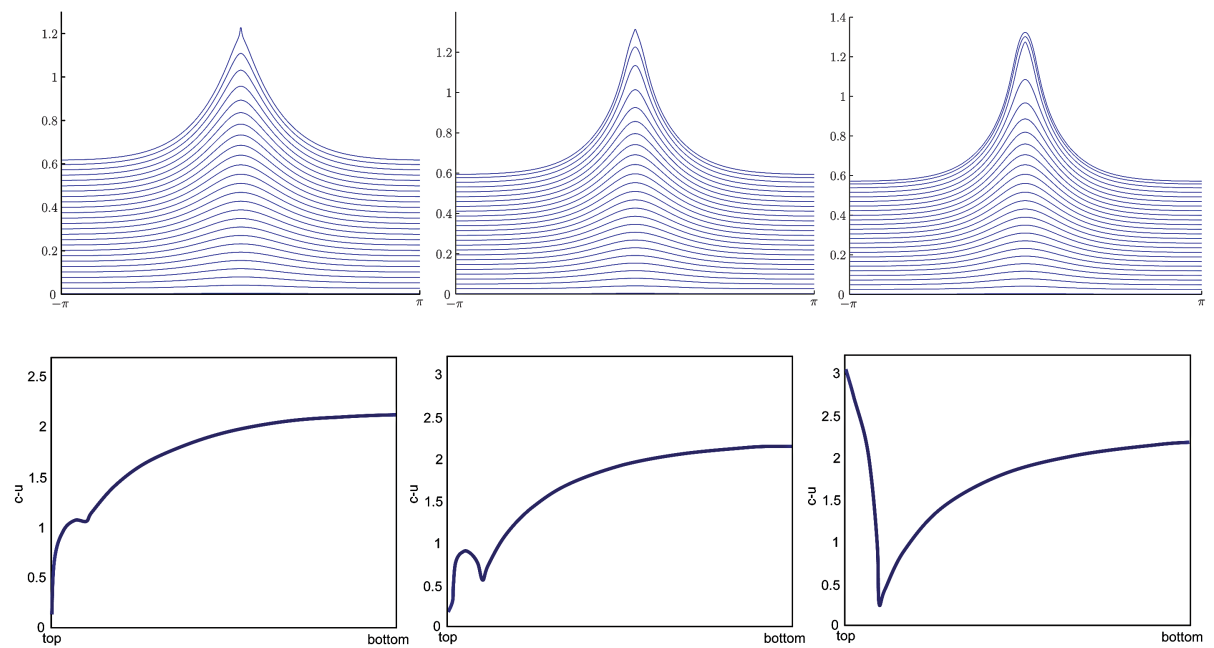

Figure 7. Top row: three waves near stagnation for a $10 \%$ shear layer with vorticity strengths $\gamma_{0}=4,10,30$. Bottom row: graphs of $c-u$ vs. $p=c y-\psi$ along the crest line from crest to bottom. Note that the almost-stagnation point in the first two cases is at the crest, but in the third case it is below the crest at the edge of the shear layer.
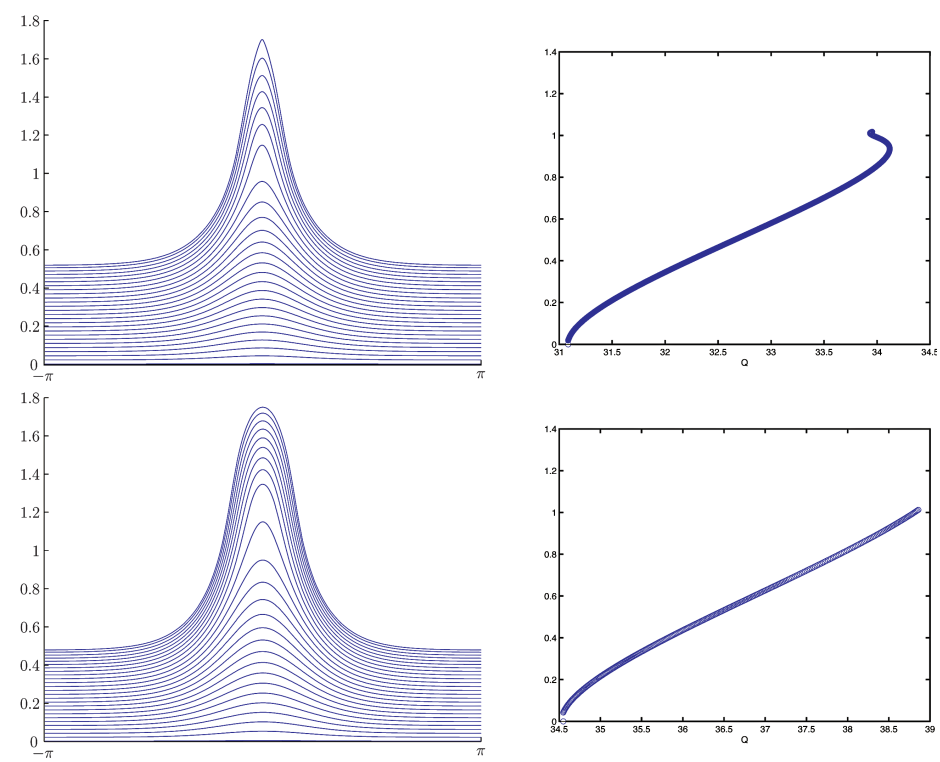

Figure 8. Left column: Two waves near stagnation with two different shear layer thicknesses: 25\% (top row) and 35\% (bottom row) and with vorticity strength $\gamma_{0}=10$. The top one stagnates at the crest, the bottom one in the interior. Right column: the bifurcation curves of $a_{\max }$ vs. $Q$ as in Figure 1. 


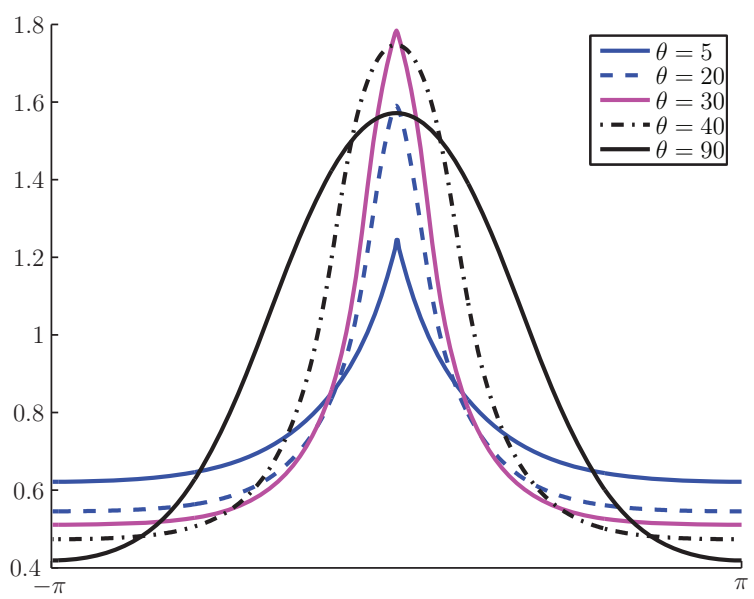

Figure 9. Profiles of waves near stagnation for five thicknesses with $\gamma_{0}=10$
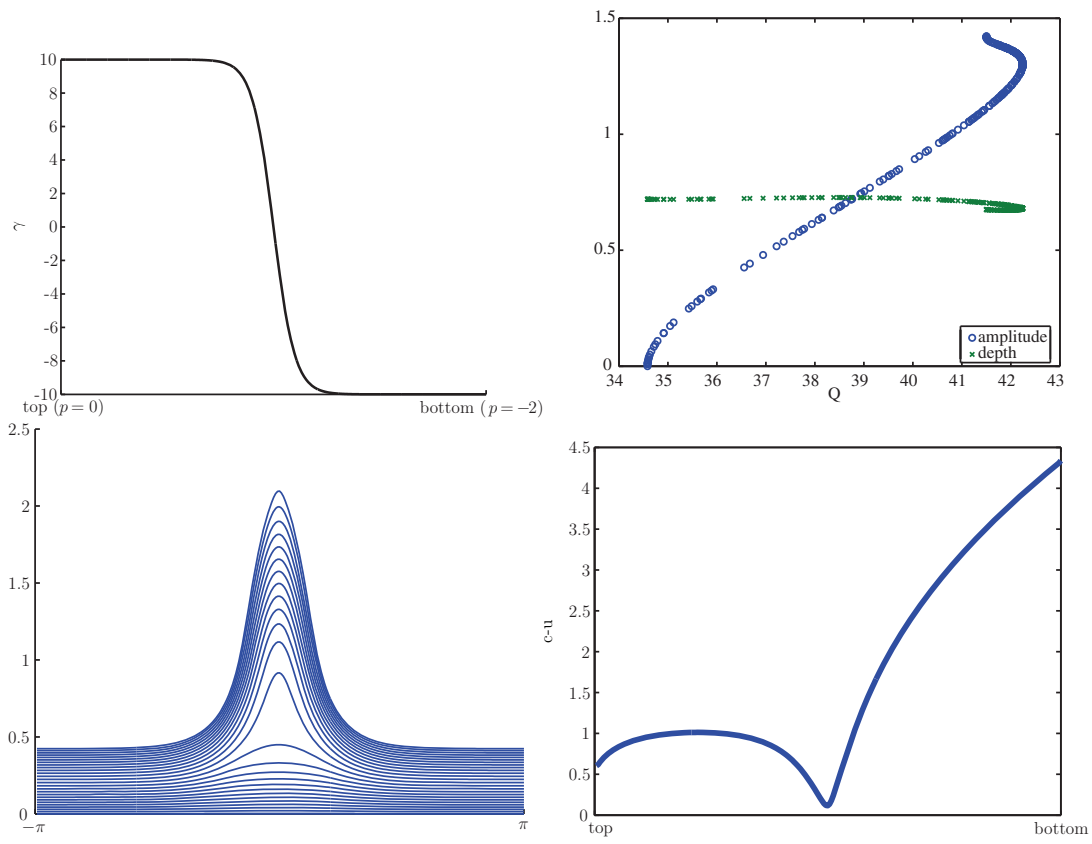

Figure 10. Continuous vorticity with internal stagnation. Clockwise from upper left: the graph of $\gamma$; the bifurcation curve (maximum amplitude and average depth as functions of $Q$ ); graph of $c-u$ vs. $p$ along the crest line from crest to bottom for the nearly stagnant wave; one period of the same wave. There is almoststagnation in the interior (where the streamlines separate). 
Several general observations can be drawn from the simulations (and from many others not shown), as follows.

(i) The amplitude increases along $\mathcal{C}$. So $a_{\max }$ occurs at the end of $\mathcal{C}$.

(ii) The almost-stagnation point (where $u \sim c$ ) is not necessarily at the crest but is always on the vertical line below the crest.

(iii) $a_{\max }$ increases as a function of $\gamma(\cdot)$ until the stagnation point is at the crest. Then it decreases.

(iv) The depth varies only slightly along $\mathcal{C}$.

(v) $a_{\max }$ increases as a function of the flux $\left|p_{0}\right|$.

(vi) The energy (or hydraulic head) $Q$ increases along $\mathcal{C}$, but then may decrease. There may be one or more turning points of $Q$.

Many of these numerical observations remain as open theoretical problems.

\section{Approximate models}

From the earliest days it was clear that waves of small amplitude could be described by much simpler approximate models. In particular, the celebrated Korteweg-deVries equation ( $\mathrm{KdV}$ ) occurs as an approximate model in several ways, not only for water waves but also for internal waves in a stratified fluid, waves in a rotating atmosphere, ion-acoustic waves in a plasma, etc.

For water waves, one can start with the irrotational Euler equations in two dimensions, assuming a free boundary $S=\{y=1+\epsilon \eta(x, t)\}$ and a finite bottom. In this case the key assumptions are that the wave has small amplitude and is unidirectional. Here $\epsilon$ is a small parameter which can be interpreted as $a / d$, where $a$ is the wave amplitude and $d$ is the average depth. After a suitable rescaling of the variables, such as writing $P=P_{a t m}-g y+\epsilon p$, one finds the system

$$
\begin{array}{cl}
u_{t}+\epsilon\left(u u_{x}+v u_{y}\right)=-p_{x}, & \epsilon v_{t}+\epsilon^{2}\left(u v_{x}+v v_{y}\right)=-p_{y}, \\
u_{x}+v_{y}=0, & u_{y}-\epsilon v_{x}=0
\end{array}
$$

together with

$$
p=\eta, v=\eta_{t}+\epsilon u \eta_{x} \quad \text { on } S=\{y=1+\epsilon \eta(x, t)\},
$$

and $v=0$ on $B=\{y=0\}$. One further rescales time $t=\tau / \epsilon$ and looks for solutions that travel to the right by introducing $\xi=x-t$. Expanding $\eta=\sum \epsilon^{k} \eta_{k}$ as well as expanding $u, v$ and $p$ formally in powers of $\epsilon$, and dropping higher-order terms, one eventually obtains for $\alpha=\partial_{\xi} \eta_{0}$ the $\mathrm{KdV}$ equation

$$
\alpha_{\tau}+\alpha_{\xi \xi \xi}+\alpha \alpha_{\xi}=0 .
$$

This equation was first derived as a water wave approximation by Boussinesq 12 and later rederived by Korteweg and de Vries 61. See 72 for the history and [55] for a clear presentation of its derivation. In the original physical variables the approximation is formally valid in a region given by $\left\{|x| \leq O\left(\epsilon^{-3 / 2}\right), t \leq O\left(\epsilon^{-3 / 2}\right)\right\}$.

There are many mathematical studies that rigorously justify this approximation. The most complete one is due to Schneider and Wayne [74, following earlier work by Kano and Nishida [56] and Craig [27]. After the appropriate scaling, Schneider and Wayne prove that an exact solution of the water wave problem splits into two "wave packets", one moving to the right and one to the left, each of which evolves independently as a solution of $\mathrm{KdV}$ for a time period $0 \leq t \leq C / \epsilon^{\frac{3}{2}}$. Here the initial 
surface displacement from a flat surface is $O(\epsilon)$ and the approximation is measured in a high Sobolev norm.

Other equations occur as limits of the irrotational water wave problem under different scaling assumptions and approximations. Some of them (in their simplest forms) are as follows.

The Boussinesq systems are not unidirectional. There are several versions but the simplest one is

$$
\alpha_{t t}-\alpha_{x x}-\left(\alpha^{2}\right)_{x x}-\alpha_{x x x x}=0 .
$$

The Camassa-Holm equation is

$$
u_{t}-u_{t x x}+3 u u_{x}=2 u_{x} u_{x x}+u u_{x x} .
$$

A distinguishing characteristic of this equation is that some of its solutions have singularities (wave breaking). The Kadomtsev-Petviashvili equation (KP) comes from the three-dimensional water wave problem. With the notation $\left(x_{1}, x_{2}, y\right)$ one assumes weak dependence on the additional horizontal variable $x_{2}$. The equation is

$$
\left\{\alpha_{t}+\alpha_{x_{1} x_{1} x_{1}}+\alpha \alpha_{x_{1}}\right\}_{x_{1}} \pm \alpha_{x_{2} x_{2}}=0 .
$$

The cubic nonlinear Schrödinger equation (NLS) occurs by slow modulation of a periodic wave. One assumes the wave is mostly unidirectional by introducing $\xi=x-c_{1} t$ but slowly modulated by another small variable $\zeta=\epsilon\left(x-c_{2} t\right)$ and $\tau=\epsilon^{2} t$. One expands in powers of $e^{i k \xi}$ with a leading term $A_{0} \exp ^{i k \xi}$, thereby eventually obtaining the NLS equation

$$
-i A_{0 \tau}+A_{0 \zeta \zeta} \pm\left|A_{0}\right|^{2} A_{0}=0 .
$$

The Davey-Stewartson system comes from the three-dimensional problem, with slow variation in $x_{2}$ and slowly modulated. It is

$$
\alpha_{x_{1} x_{1}}+\alpha_{x_{2} x_{2}}+\left(|\beta|^{2}\right)_{x}=0, \quad i \beta_{t}+\beta_{x_{1} x_{1}}-\beta_{x_{2} x_{2}}+|\beta|^{2} \beta+\alpha_{x_{1}} \beta=0 .
$$

It is remarkable that all of these equations are "completely integrable", in spite of the fact that complete integrability is an extremely special phenomenon and the full water wave problem itself is apparently not completely integrable. The solutions of each of these special equations can be described completely (but totally explicitly) in terms of the spectrum of an associated linear operator. This spectrum evolves very simply, so that the evolution of the nonlinear problem reduces to solving an inverse spectral or inverse scattering problem, which means that one has to find a potential that leads to given spectral or scattering data. Some publications on these topics are [2, 11, 19, 31, 55, 74].

\section{EXTENSIONS AND OPEN PROBLEMS}

There are many additional directions that have been taken in the theory of steady water waves with vorticity.

7.1. Location of the stagnation points. If the vorticity function $\gamma(\cdot)$ is nonpositive, in the context of Theorem 4.1, it is known [80] that $P(x, y) \geq P_{a t m}$ everywhere and that the horizontal velocity $u(x, y)$ is maximized at the crest. Furthermore, if $Q$ is bounded for waves in $\mathcal{C}$, then there exists an "extreme" wave such that $\max u=c$, which is obtained as a weak limit of nonextreme waves [81. If $\gamma \leq 0, \gamma^{\prime} \geq 0, \gamma(0)<0$, then $Q$ is indeed bounded along $\mathcal{C}$ and the extreme 
wave has a stagnation point at the crest, where there is either a corner of $120^{\circ}$ or a horizontal tangent.

7.2. Pressure. In the irrotational case $\gamma=0$, the pressure $P(x, y)$ strictly increases with depth and strictly decreases horizontally away from the crest line 25]. There is numerical evidence but as yet only a conjecture that such behavior is not always true in the presence of vorticity. If $u<c$, all the particle trajectories in an irrotational flow are nonclosed looping orbits unless there is a background current 25, 17].

7.3. Higher modes. There are also steady waves with more than one crest (local maximum) and trough (local minimum) per period [71. Such waves are generated by local bifurcation from solutions of (4.6) of the form $m=M(p) \cos (k 2 \pi q / L)$ with $k \geq 2$. They have been much less studied than the case $k=1$.

7.4. Variational characterizations. There are several variational (Hamiltonian or Lagrangian) methods that characterize irrotational steady water waves. They go back to Friedrichs [45] in 1933 and Zakharov 90] in 1968. Zakharov's method works for a time-dependent, irrotational flow. It uses the velocity potential $\phi$ defined by $\nabla \phi=\mathbf{u}$, which exists since $\nabla \times \mathbf{u}=\mathbf{0}$, so that $\Delta \phi=0$. Then the surface $S$ (given by $\eta$ ) and the restriction $\xi=\left.\phi\right|_{S}$ are treated as the new unknowns. The Hamiltonian (in two dimensions with period $2 \pi$ say) then is

$$
\mathcal{H}(\eta, \xi)=\frac{g}{2} \int_{0}^{2 \pi} \eta^{2} d x+\frac{1}{2} \int_{0}^{2 \pi} \xi \cdot G(\eta) \xi d x,
$$

where $G(\eta)$ is the Dirichlet-Neumann operator defined by $G(\eta) \xi=-\eta_{x} \phi_{x}+\phi_{y}$. This has been a useful formulation. It was used for instance by Craig and Sulem for purposes of simulation 30 .

Unfortunately, no Hamiltonian formulation is known for waves that are both time-dependent and rotational, other than for approximate models. But for steady 2D periodic waves with a strictly monotone vorticity function $\left(\gamma^{\prime} \neq 0\right)$, there is the following formulation. For such a $\gamma$, choose $F$ so that $\gamma^{-1}=F^{\prime}$. Define the "energy"

$$
\mathcal{E}(\eta, \psi)=\int_{0}^{L} \int_{-d}^{\eta(x)}\left\{\frac{1}{2}|\nabla \psi|^{2}+g y-F(-\Delta \psi)\right\} d y d x,
$$

mass $m=\iint d y d x$ and horizontal momentum $M=\iint \partial_{y} \psi d y d x$. These three functionals are invariants of the time-dependent problem. Then any critical point of $\mathcal{E}$, subject to periodicity and to the constraints that $m$ and $M$ are constants, is a steady water wave of period $L$ with vorticity function $\gamma[20$. Furthermore, let $\gamma$ be positive and decreasing with depth $\left(\gamma^{\prime}<0\right)$. Then the steady water wave is linearly stable if either the free surface is unperturbed or the velocity on the surface is perturbed only normally [24].

7.5. Infinite depth. At very large depths (think of the ocean) we expect essentially no motion. Thus if we idealize a deep ocean as occupying a region $\{-\infty<y \leq \eta(x, t)\}$, the boundary condition is that $\mathbf{u} \rightarrow \mathbf{0}$ as $y \rightarrow-\infty$. This is different from the finite-depth case where there is motion tangent to the bottom surface. In some ways infinite depth is the easier case, as in Gerstner's example [47, but the loss of compactness is a serious mathematical complication. The natural bifurcation parameter is the wave speed $c$. The transformed problem for the height $h(q, p)$ is (4.2) and (4.5) but with (4.4) replaced by $h_{q} \rightarrow 0, h_{p} \rightarrow 1 / c$ as $p \rightarrow-\infty$ 
and with (4.3) replaced by $1+h_{q}^{2}+2 g h h_{p}^{2}=0$. Hur [51, 53] has proven an analogue of Theorem 4.1 in case $\gamma(p)=O\left(|p|^{-2-\epsilon}\right)$ for large $|p|$ and some $\epsilon>0$. Her conclusion is that either there exist waves approaching stagnation or the wave speeds $c \rightarrow \infty$. There is a stronger conclusion if $\gamma$ remains of one sign and is monotone.

7.6. Solitary waves. This is the problem (in two dimensions, say) where instead of the periodicity the surface should become flat at infinity: $\eta(x)$ tends to a constant and $v \rightarrow 0$ as $x \rightarrow \pm \infty$. It can be viewed formally as the limit as the period of the wave tends to infinity. Much effort has been expended to prove the existence of exact solitary water waves. For zero vorticity the rigorous theory goes back to Lavrentiev in 1954, Ter-Krikorov, Friedrichs and Hyers, and Beale. See the references in [8]. Irrotational large-amplitude solitary waves were constructed by Amick and Toland [5]. On the other hand, in the presence of an arbitrary vorticity function $\gamma(\cdot)$, Hur [52] used a Nash-Moser construction, a generalization of Beale's, to prove the existence of a curve of small-amplitude solitary waves. Groves and Wahlén [49] give a similar result using the method of spatial dynamics [7].

7.7. Symmetry. It has been shown under very general conditions that all periodic and solitary water waves, not just those constructed as in Theorem 4.1, must a priori be symmetric around their crests. The most effective proofs are based on the method of moving planes. The irrotational case was proven by Craig and Sternberg [29] while the rotational case is due in its greatest generality to Constantin, Ehrnström and Wahlén [18; other recent contributors are Escher and Hur.

7.8. Surface tension. Of course, some surface tension always occurs at the airwater interface, but for large-scale waves as in the ocean, its effect is small. A wave with gravity $g>0$ and surface tension $\sigma>0$ is called a capillary-gravity wave. Instead of (2.1), the boundary conditions are

$$
P=P_{a t m}-\sigma \kappa, \quad v=\eta_{t}+u \eta_{x} \quad \text { on } S,
$$

where $\kappa$ is the curvature and $\sigma$ is usually assumed to be a constant. For capillarygravity waves with vorticity, it was first observed by Wahlén 83 that there is local bifurcation and that the lowest eigenvalue $(k=1)$ of the bifurcation problem is either simple or double. Walsh [84 has analyzed in detail the possible behavior near double eigenvalues using the Liapunov-Schmidt method, proving that there can be as many as four separate smooth curves emanating from the bifurcation point and that each of these curves is global.

7.9. Stratified fluids. Stratification is a common feature of ocean waves due to variations in salinity and temperature, which have a major effect on the waves. In the simplified context of this article, it means that the density $\rho$ is a variable. We continue to assume that the fluid is inviscid and incompressible. Incompressibility and conservation of mass imply the pair of equations

$$
\nabla \cdot \mathbf{u}=0, \quad \frac{\partial \rho}{\partial t}+\mathbf{u} \cdot \nabla \rho=0 .
$$

Conservation of momentum is almost the same as before:

$$
\frac{\partial \mathbf{u}}{\partial t}+(\mathbf{u} \cdot \nabla) \mathbf{u}+\frac{1}{\rho} \nabla P=\mathbf{F} .
$$

The boundary conditions are exactly as stated in Section 1 . Then for a steady wave depending on $(x-c t, y)$, and with $x-c t$ replaced by $x$ as before, the density $\rho$ 
depends on the stream function: $\rho=\rho(-\psi(x, y)+c y)$ due to (7.1). Then there exist steady waves of small and large amplitude, obtained by local and global bifurcation [85]. The proof follows the same lines as in Section 4 but with some major complications. There is also an extensive literature on internal waves in a stratified fluid [10, 6].

7.10. Overhanging waves. Breakers at the beach are overhanging in the sense that the height $\eta$ of the wave is multi-valued. Do there exist steady waves that are overhanging? They cannot exist if the vorticity vanishes. But for positive vorticity there is numerical evidence that they do exist [35.

7.11. Three-dimensional steady waves. The existence of steady waves that are truly three-dimensional is another mostly open problem. Consider irrotational gravity waves without surface tension. Let $\phi$ be the velocity potential, so that $\Delta \phi=0$. Even local bifurcation is very difficult because of the phenomenon of "small divisors". Indeed, Airy's dispersion relation (see Section 2) now takes the form

$$
g|\mathbf{k}| \tanh (|\mathbf{k}| d)=(\mathbf{c} \cdot \mathbf{k})^{2},
$$

where the wave speed and direction are $\mathbf{c} \in \mathbb{R}^{2}$, the Fourier variable to $\mathbf{x} \in \mathbb{R}^{2}$ is $\mathbf{k} \in \mathbb{R}^{2}$ and the depth is $d$. That is, the steady wave in the physical variables is $\mathbf{u}(\mathbf{x}-\mathbf{c} t, y) \in \mathbb{R}^{3}$, where $y$ is the vertical variable and we are looking for waves of the form $\exp (i \mathbf{k} \cdot \mathbf{x})$. The dispersion relation (7.3) has infinitely many solutions $\mathbf{k}$ that have accumulation points. When local bifurcation is attempted, this leads to eigenvalues $\lambda$ that accumulate at $\lambda=0$, which prevents the use of the implicit function theorem. Numerical computations suggest the existence of hexagonal waves of small amplitude 28. The first important theoretical result on this problem is due to Iooss and Plotnikov [54]. On the other hand, it is known that surface tension moderates the difficulty; there are several existence theorems for capillary-gravity waves in $3 \mathrm{D}$, but we omit the references.

7.12. Instability. If we perturb a steady wave at time $t=0$, does the (timedependent) wave at all later times remain near (a translate of) the steady wave? The steady wave would then be called "stable", and otherwise, "unstable". Consider the irrotational case. Benjamin and Feir 9 showed formally that, in a small-amplitude approximation, there is a "sideband" instability, meaning that the perturbation has a slightly different period from the steady wave. A rigorous proof of this linear instability was given by Bridges and Mielke [13. Once again, it is known that surface tension has a stabilizing influence. Without surface tension, there is also some discussion of the stability problem in the rotational case in 24. Lin 67] has succeeded in proving the linearized instability of some solitary waves of large amplitude.

\section{ABout the AUThor}

Walter Strauss is the L. Herbert Ballou University Professor at Brown University. He was a Guggenheim Fellow and is a SIAM Fellow. 


\section{REFERENCES}

1. G. B. Airy, Tides and waves, Encyclopedia Metropolitana 5 (1845), 241-396.

2. B. Alvarez-Samaniego and D. Lannes, Large time existence for $3 D$ water-waves and asymptotics, Invent. Math. 171 (2008), 485-541. MR2372806 (2009b:35324)

3. D. Ambrose, Well-posedness of vortex sheets with surface tension, SIAM J. Math. Anal. 35 (2003), 211-244. MR2001473 (2005g:76006)

4. D. Ambrose and N. Masmoudi, Well-posedness of $3 D$ vortex sheets with surface tension, Commun. Math. Sci. 5 (2007), 391-430. MR2334849 (2008g:76010)

5. C. Amick and J. F. Toland, On solitary water-waves of finite amplitude, Arch. Rational Mech. Anal. 76 (1981), 9-95. MR629699 (83b:76017)

6. C. Amick and R. Turner, Small internal waves in two-fluid systems, Arch. Rational Mech. Anal. 108 (1989), 111-139. MR.1011554 (90h:76037)

7. _ Center manifolds in equations from hydrodynamics, Nonlinear Diff. Eq. Appl. 1 (1994), 47-90. MR1273343 (95d:58115)

8. J. T. Beale, The existence of solitary water waves, Comm. Pure Appl. Math. 5 (1977), 237275. MR0445136 (56:3480)

9. T. B. Benjamin and J. E. Feir, The disintegration of wavetrains in deep water, J. Fluid Mech. 27 (1967), 417-430.

10. J. Bona, D. Bose, and R. Turner, Finite-amplitude steady waves in stratified fluids, J. Math. Pures Appl. 62 (1983), 389-439. MR735931 (85e:76056)

11. J. Bona, T. Colin, and D. Lannes, Long wave approximations for water waves, Arch. Rat. Mech. Anal. 178 (2005), 373-410. MR2196497 (2007a:76012)

12. M. J. Boussinesq, Essai sur la théorie des eaux courantes, Mémoirs présentés par divers savants à l'Acad. des Sciences Inst. France (série 2) 23 (1877), 1-680.

13. T. Bridges and A. Mielke, A proof of the Benjamin-Feir instability, Arch. Rat. Mech. Anal. 133 (1995), 145-198. MR.1367360 (97c:76028)

14. B. Buffoni and J. Toland, Analytic theory of global bifurcation, Princeton, 2003. MR 1956130 (2004b:47117)

15. A. Cauchy, Théorie de la propagation des ondes à la surface d'un fluide pésant, Oeuvres Complètes 1 (1827).

16. A. Constantin, On the deep water wave motion, J. Phys. A 34 (2001), 1405-1417. MR.1819940 (2002b:76010)

17. The trajectories of particles in Stokes waves, Invent. Math. 166 (2006), 523-535. MR 2257390 (2007j:35240)

18. A. Constantin, M. Ehrnström, and E. Wahlén, Symmetry of steady periodic gravity water waves with vorticity, Duke Math. J. 140 (2007), 591-603. MR2362244 (2009c:35359)

19. A. Constantin and D. Lannes, The hydrodynamical relevance of the Camassa-Holm and Degasperis-Procesi equations, Arch. Ration. Mech. Anal. 192 (2009), 165-186. MR2481064 (2010f:35334)

20. A. Constantin, D. Sattinger, and W. Strauss, Variational formulations for steady water waves with vorticity, J. Fluid Mech. 548 (2006), 151-168. MR2264220 (2008b:76018)

21. A. Constantin and W. Strauss, Periodic traveling gravity water waves with discontinuous vorticity (preprint 2010).

22. __ Exact steady periodic water waves with vorticity, Comm. Pure Appl. Math. 57 (2004), no. 4, 481-527. MR2027299 (2004i:76018)

23. _ Rotational steady water waves near stagnation, Phil. Trans. Roy. Soc 365 (2007), 2227-2239. MR2329144 (2008j:76013)

24. Stability properties of steady water waves with vorticity, Comm. Pure Appl. Math. 60 (2007), 911-950. MR2306225 (2009b:35257)

25. _ Pressure beneath a Stokes wave, Comm. Pure Appl. Math. 63 (2010), 533-557. MR2604871

26. D. Coutand and S. Shkoller, Well-posedness of the free-surface incompressible Euler equations with or without surface tension, J. Amer. Math. Soc. 20 (2007), 829-930. MR2291920 (2008c:35242)

27. W. Craig, An existence theory for water waves and the Boussinesq and Korteweg-de Vries scaling limits, Comm. PDE 10 (1985), 787-1003. MR795808 (87f:35210) 
28. W. Craig and D. Nicholls, Traveling gravity water waves in two and three dimensions, Eur. J. Mech. B Fluids 21 (2002), 615-641. MR1947187(2004b:76018)

29. W. Craig and P. Sternberg, Symmetry of solitary waves, Comm. P.D.E. 13 (1988), 603-633. MR 919444 (88m:35132)

30. W. Craig and C. Sulem, Numerical simulation of gravity waves, J. Comp. Phys. 108 (1993), 73-83. MR:1239970 (94h:76064)

31. W. Craig, C. Sulem, and P.-L. Sulem, Nonlinear modulation of gravity waves: A rigorous approach, Nonlinearity 5 (1992), 497-522. MR1158383 (93k:76012)

32. W. Craig and C. E. Wayne, Mathematical aspects of surface waves on water, Russian Math. Surveys 62 (2007), 453-473. MR2355420(2009h:76001)

33. A. Craik, The origins of water wave theory, Annu. Rev. Fluid Mech. 36 (2004), 1-28. MR 2062306 (2005a:01012)

34. M. Crandall and P. Rabinowitz, Bifurcation from simple eigenvalues, J. Funct. Anal. 8 (1971), 321-340. MR0288640 (44:5836)

35. A. Teles da Silva and D. Peregrine, Steep, steady surface waves on water of finite depth with constant vorticity, J. Fluid Mech. 195 (1988), 281-302. MR985439(90a:76061)

36. A. Dalmedico, La propagation de ondes en eau profonde et ses développements mathématiques, The History of Modern Mathematics, D. Rowe and J. McCleary, eds., 2 (1989), 129-168.

37. E. N. Dancer, Global solution branches for positive mappings, Arch. Rat. Mech. Anal. 52 (1973), 181-192. MR0353077 (50:5563)

38. O. Darrigol, The spirited horse, the engineer, and the mathematician: Water waves in nineteenth-century hydrodynamics, Arch. Hist. Exact Sci. 58 (2003), 21-95. MR 2020055 (2004k:76002)

39. W Worlds of flow, Oxford, 2005. MR2178164 (2006j:76001)

40. F. Dias and G. Iooss, Water-waves as a spatial dynamical system, Handbook of mathematical fluid dynamics II, North-Holland, Amsterdam, 2003, pp. 443-499. MR.1984157 (2004g:76021)

41. M.-L. Dubreil-Jacotin, Sur la détermination rigoureuse des ondes permanentes périodiques d'ampleur finie, J. Math. Pures Appl. 13 (1934), 217-291.

42. L. Euler, General principles of the motion of fluids, Hist. de l'Acad. de Berlin 11 (1755), 274-315.

43. C. Fefferman, Existence and smoothness of the Navier-Stokes equation. The millennium prize problems, Clay Math. Inst., Cambridge, MA, 2006, pp. 57-67. MR2238274

44. L. E. Fraenkel, An introduction to maximum principles and symmetry in elliptic problems, Cambridge, 2000. MR.1751289 (2001c:35042)

45. K. O. Friedrichs, Uber ein minimumproblem für potential strömung mit freien rand, Math. Ann. 109 (1933), 60-82.

46. P. Germain, N. Masmoudi and J. Shatah, Global solutions for the gravity water waves equation in dimension 3, C. R. Math. Acad. Sci. Paris 347 (2009), 897-902 (and preprint 2009). MR.2542891

47. F. Gerstner, Theorie der wellen, Abhand. Koen. Boehmischen Gesel. Wiss., Prague, 1802.

48. M. Groves, Steady water waves, J. Nonl. Math. Phys. 11 (2004), 435-460. MR.2097656 (2006a:76014)

49. M. Groves and E. Wahlén, Small-amplitude Stokes and solitary waves with an arbitrary distribution of vorticity, Phys. D 237 (2008), 1530-1538. MR2454604

50. T. Healey and H. Simpson, Global continuation in nonlinear elasticity, Arch. Rat. Mech. Anal. 143 (1998), 1-28. MR:1643646 (99h:73021)

51. V. M. Hur, Global bifurcation of deep-water waves, SIAM J. Math. Anal. 37 (2006), 14821521. MR2215274 (2007e:76025)

52. _ Exact solitary water waves with vorticity, Arch. Rational Mech. Anal. 188 (2008), 213-244. MR2385741 (2010b:76019)

53. _ Stokes waves with vorticity (preprint 2009).

54. G. Iooss and P. Plotnikov, Small divisor problem in the theory of three-dimensional water gravity waves, Memoirs Amer. Math. Soc. (2009). MR2529006

55. R. S. Johnson, A modern introduction to the mathematical theory of water waves, Cambridge University Press, 1997. MR1629555 (99m:76017)

56. T. Kano and T. Nishida, Sur les ondes de surface de l'eau avec une justification mathématique des équations des ondes en eau peu profonde, J. Math. Kyoto Univ. 19 (1979), 335-370. MR:545714 (82d:76012) 
57. G. Keady and J. Norbury, On the existence theory for irrotational water waves, Math. Proc. Cambridge Phil. Soc. 83 (1978), 137-157. MR0502787 (58:19712)

58. S. Klainerman, Global existence of small amplitude solutions to nonlinear Klein-Gordon equations in four space-time dimensions, Comm. Pure Appl. Math. 38 (1985), 631-641. MR803252 (87e:35080)

59. Joy Ko and W. Strauss, Large-amplitude steady rotational water waves, Eur. J. Mech. B Fluids 27 (2007), 96-109. MR2389493 (2009b:76014)

60. _ Effect of vorticity on steady water waves, J. Fluid Mech. 608 (2008), 197-215. MR:2439751 (2009f:76023)

61. D. Korteweg and G. de Vries, On the change of form of long waves advancing in a rectangular canal, Phil. Mag. 39 (1895), 422-443.

62. Yu. Krasovskii, On the theory of steady-state waves of finite amplitude, USSR Comput. Math. and Math. Phys. 1 (1961), 996-1018. MR0138284 (25:1731)

63. D. Lannes, Well-posedness of the water-waves equations, J. Amer. Math. Soc. 18, 605-654. MR 2138139 (2005m:76021)

64. J. Leray, Sur le mouvement d'un liquide visqueux emplissant l'espace, Acta Math. 63 (1934), 193-248. MR1555394

65. T. Levi-Civita, Determinazione rigorosa delle onde irrotazionali periodiche in acqua profonda, Rend. Accad. Lincei 33 (1924), 141-150.

66. G. Lieberman and N. Trudinger, Nonlinear oblique boundary value problems for nonlinear elliptic equations, Trans. Amer. Math. Soc. 295 (1986), 509-546. MR833695 (87h:35114)

67. Zhiwu Lin, On linear instability of $2 D$ solitary water waves, Inter. Math. Res. Not. 2009, no. 7, 1247-1303. MR2495304(2010f:35310)

68. H. Lindblad, Well posedness for the motion of a compressible liquid with free surface boundary, Comm. Math. Phys. 260 (2005), 319-392. MR2177323 (2006g:35219)

69. W Well-posedness for the motion of an incompressible liquid with free surface boundary, Ann. of Math. (2) 162 (2005), 109-194. MR2178961 (2006g:35293)

70. A. Nekrasov, On steady waves, Izv. Ivanovo-Voznesenk. Politekhn. 3 (1921).

71. H. Okamoto and M. Shoji, The mathematical theory of permanent progressive water-waves, Advanced Series in Nonlinear Dynamics, vol. 20, World Scientific Publishing Co. Inc., River Edge, NJ, 2001. MR1869386(2003h:76016)

72. R. Pego and M. Weinstein, Convective linear stability of solitary waves for Boussinesq equations, Stud. in Appl. Math. 99 (1997), 311-375. MR1477120 (99a:35219)

73. P. Rabinowitz, Some global results for nonlinear eigenvalue problems, J. Funct. Anal. 7 (1971), 487-513. MR0301587 (46:745)

74. G. Schneider and C. E. Wayne, The long-wave limit for the water wave problem: The case of zero surface tension, Comm. Pure Appl. Math. 53 (2000), 1475-1535. MR.1780702 (2002c:76025a)

75. B. Schweizer, On the three-dimensional Euler equations with a free boundary subject to surface tension, Ann. Inst. H. Poincaré Anal. Non Linéaire 22 (2005), 753-781. MR2172858 (2006g:35222)

76. J. Shatah, Normal forms and quadratic nonlinear Klein-Gordon equations, Comm. Pure Appl. Math. 38 (1985), 685-696. MR803256 (87b:35160)

77. J. Shatah and C. Zeng, Geometry and a priori estimates for free boundary problems of the Euler equation, Comm. Pure Appl. Math. 61 (2008), 698-744. MR2388661(2010b:76014)

78. G. Stokes, On the theory of oscillatory waves, Trans. Camb. Phil. Soc. 8 (1847), 441-455.

79. J. F. Toland, Stokes waves, Topol. Methods Nonlinear Anal. 7 (1996), no. 1, 1-48. MR.1422004 $(97 \mathrm{j}: 35130)$

80. E. Varvaruca, On some properties of travelling water waves with vorticity, SIAM J. Math. Anal. 39 (2008), 1686-1692. MR2377294 (2008m:76018)

81. __ On the existence of extreme waves and the Stokes conjecture with vorticity, J. Diff. Eq. 246 (2009), 4043-4076. MR2514735

82. E. Wahlén, A note on steady gravity waves with vorticity, Int. Math. Res. Not. 2005, 389-396. MR2130838 (2006b:76015)

83. $\quad$ Steady periodic capillary-gravity waves with vorticity, SIAM J. Math. Anal. 38 (2006), 921-943. MR2262949 (2007g:35196)

84. S. Walsh, Steady periodic gravity waves with surface tension (preprint 2010). 
85. _ Stratified steady periodic water waves, SIAM J. Math. Anal. 41, (2009), 1054-1105. MR 2529956

86. Sijue $\mathrm{Wu}$, Well-posedness in Sobolev spaces of the full water wave problem in 2-D, Invent. Math. 130 (1997), 39-72. MR:1471885 (98m:35167)

87. W Well-posedness in Sobolev spaces of the full water wave problem in 3-D, J. Amer. Math. Soc. 12 (1999), 445-495. MR1641609 (2001m:76019)

88. _ Almost global wellposedness of the 2-D full water wave problem, Invent. Math. $\mathbf{1 7 7}$ (2009), 45-135. MR2507638

89. G Global wellposedness of the 3-D full water wave problem (preprint 2009).

90. V. E. Zakharov, Stability of periodic waves of finite amplitude on the surface of a deep fluid, J. Appl. Mech. Tech. Phys. 2 (1968), 190-194.

91. P. Zhang and Z. Zhang, On the free boundary problem of three-dimensional incompressible Euler equations, Comm. Pure Appl. Math. 61 (2008), 877-940. MR2410409 (2009h:35461)

Department of Mathematics and Lefschetz Center for Dynamical Systems, Brown University, Providence, Rhode Island

E-mail address: wstrauss@math.brown.edu 